\title{
MicroRNA-214 Affects Fibroblast Differentiation of Adipose-Derived Mesenchymal Stem Cells by Targeting Mitofusin-2 during Pelvic Floor Dysfunction in SD Rats with Birth Trauma
}

Jing Wu Jiang $\mathrm{Li}^{\mathrm{b}}{ }^{\mathrm{a}}$ Wei-Kang Chen ${ }^{\mathrm{b}}$ Shang Liu ${ }^{\mathrm{b}}$ Jian-He Liu ${ }^{\mathrm{b}}$ Jing-Song Zhang Ke-Wei Fang ${ }^{b}$

aDepartment of Biochemistry and Molecular Biology, School of Basic Medical Sciences, Kunming Medical University, Kunming, 'D Department of Urology, The Second Affiliated Hospital of Kunming Medical University, Kunming, P.R. China 